\title{
Some differential properties of grand generalized Sobolev-Morrey spaces
}

\author{
Rovshan F. Babayev
}

Received: 23.01.2020 / Revised: 03.08.2020 / Accepted: 02.10.2020

Abstract. In this paper we introduce a grand generalized Sobolev-Morrey spaces. Also, with the help of integral representations we study differential properties of functions from this spaces.

Keywords. Grand generalized Sobolev-Morrey spaces, embedding theorems, integral representation, Hölder condition.

Mathematics Subject Classification (2010): 46E35, 46E30, 26D15

\section{Introduction and preliminary notes}

This paper is devoted to investigations of embedding theorems for the grand generalized Sobolev-Morrey spaces

$$
\bigcap_{i=0}^{n} L_{p^{i}, \varkappa, a}^{<l^{i}>}(G)
$$

where $G \subset \mathbb{R}^{n}$ is a bounded domain, $1<p^{i}<\infty ; l^{i} \in N_{0}^{n}(i=0,1,2, \ldots, n)$, $l_{j}^{0} \geq 0,(j=1,2, \ldots, n) ; l_{j}^{i} \geq 0,(i=0,1,2, \ldots, n), l_{i}^{i}>0$ are non-negative integers $(i=0,1,2, \ldots, n) ; a \in[0,1] ; \varkappa \in(0, \infty)^{n}$. First we introduce a grand generalized Sobolev-Morrey, and on the based of the method of integral representation we study some differential properties of functions, defined on $n$-dimensional domains satisfying flexible $\varphi$ - horn condition (see, [12]).

Note that the grand Lebesgue spaces $L_{p)}(G)$ for a measurable set $G \subset \mathbb{R}^{n}$ of finite Lebesgue measure were introduced in the work of T. Iwaniec and C. Sbordone in [3]. After a vast amount of research about small Lebesgue-Morrey space, grand and small Sobolev spaces, grand grand Lebesgue-Morrey space, grand grand and small small Sobolev-Morrey spaces, grand grand Nikolskii-Morrey spaces, and grand Sobolev-Morrey spaces with dominant mixed derivatives (with different norms) has been done by many mathematicians (see,e.g. $[2,4-8,10,11,13-21])$.

Let $G \subset \mathbb{R}^{n}$ is a bounded domain, $t>0$, and let for all $x \in \mathbb{R}^{n}$

$$
G_{t^{\varkappa}}(x)=G \cap\left\{y:\left|y_{j}-x_{j}\right|<\frac{1}{2} t^{\varkappa_{j}}, j=1,2, \ldots, n\right\} .
$$

R.F. Babayev

Mingechevir State University, Mingechevir, Azerbaijan.

E-mail: aliknajafov@gmail.com 
Definition 1.1 We denote by $\bigcap_{i=0}^{n} L_{p^{i}, \varkappa, a}^{<l^{i}>}(G)$ the space of locally summable functions $f$ on $G$ having weak derivatives $D^{l^{i}} f(i=0,1, \ldots, n)$ with the finite norm

$$
\|f\|_{\bigcap_{i=0}^{n} L_{\left.p^{i}\right), \varkappa, a}^{<l^{i}>}(G)}=\sum_{i=0}^{n}\left\|D^{l^{i}} f\right\|_{\left.p^{i}\right), \varkappa, a ; G} .
$$

Here

$$
\begin{gathered}
\|f\|_{\left.p^{i}\right), \varkappa, a ; G}=\|f\|_{L_{\left.p^{i}\right), \varkappa, a}(G)} \\
=\sup _{\substack{0<t \leq d_{0}, x \in G, 0<\in \varepsilon<p-1}}\left(\frac{1}{t^{|\varkappa| a}} \frac{\varepsilon}{\left|G_{t^{\varkappa}}(x)\right|} \int_{G_{t^{\varkappa}}(x)}|f(y)|^{p^{i-\varepsilon}} d y\right)^{\frac{1}{p^{i}-\varepsilon}}, \\
\end{gathered}
$$

and $d_{0}=\operatorname{diam} G$.

Note that the space $L_{p), a, \varkappa}(G)$ defined and studied in [13], and $\bigcap_{i=0}^{n} L_{\left.p^{i}\right), \varkappa, a}(G)$, in the case $l^{0}=(0, \ldots, 0), l^{i}=\left(0, \ldots, 0, l_{i}, 0, \ldots, 0\right), p^{i}=p(i=0,1, \ldots, n)$ coincides with the grand Sobolev-Morrey space $W_{p), a, \varkappa}^{l}(G)$ introduced and studied in [13].

We give some properties of the spaces $\bigcap_{i=0}^{n} L_{\left.p^{i}\right), a, \varkappa}^{<l>}(G)$.

1.The following embedding holds:

$$
\bigcap_{i=0}^{n} L_{\left.p^{i}\right), \varkappa, a}^{<l^{i}>}(G) \hookrightarrow \bigcap_{i=0}^{n} L_{p^{i}}^{<l^{i}>}(G),
$$

i.e.,

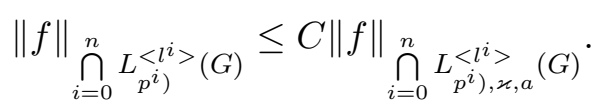

Norm in grand generalized Sobolev space $\bigcap_{i=0}^{n} L_{p^{i}}^{<l^{i>}}(G)$ defined as:

$$
\begin{gathered}
\|f\|_{\bigcap_{i=0}^{n} L_{\left.p^{i}\right)}^{<i>}(G)}=\sum_{i=0}^{n}\left\|D^{l^{i}} f\right\|_{\left.p^{i}\right), G}, \\
\|f\|_{\left.p^{i}\right), G}=\|f\|_{L_{\left.p^{i}\right)}(G)}=\sup _{0<\varepsilon<p^{i}-1}\left(\frac{\varepsilon}{|G|} \int_{G}|f(x)|^{p^{i}-\varepsilon} d x\right)^{\frac{1}{p^{i}-\varepsilon}}
\end{gathered}
$$

2. The spaces $\bigcap_{i=0}^{n} L_{\left.p^{i}\right), \varkappa, a}^{<l^{i>}}(G)$ is complete;

3. $\|f\|_{\bigcap_{i=0}^{n} L_{\left.p^{i}\right), \varkappa, 0}^{<i>}(G)}=\|f\|_{\bigcap_{i=0}^{n} L_{\left.p^{i}\right)}^{<l^{i}>}(G)}^{n}$.

To prove the main theorems, we need some auxiliary inequalities given in the lemma below. Let $\psi(\cdot, y, z) \in C_{0}^{\infty}\left(\mathbb{R}^{n}\right)$ be such that

$$
S(\psi)=\operatorname{supp} \psi \subset I_{1}=\left\{x:\left|x_{j}\right|<\frac{1}{2}, j=1,2, \ldots, n\right\},
$$


and let $0<T \leq 1, \lambda=\left(\lambda_{1}, \ldots, \lambda_{n}\right), \lambda_{j}>0(j=1,2, \ldots, n)$ and put

$$
V=\bigcup_{0<t \leq T}\left\{y:\left(\frac{y}{t^{\lambda}}\right) \in S(\psi)\right\} .
$$

Clearly, $V \subset I_{T^{\lambda}}=x ;\left|x_{j}\right|<\frac{1}{2} T_{j}^{\lambda}, j=1,2, \ldots, n$, and let $U$ be an open set contained in the domain $G$; henceforth we always assume that $U+V \subset G$, and put $G_{T^{\varkappa}}(U)=$ $U+I_{T^{\varkappa}(x)} \cap G$.

Obviously, if $0<\varkappa_{j} \leq \lambda_{j}(j=1,2, \ldots, n)$, then $I_{T^{\lambda}} \subset I_{T^{\varkappa}}$ and therefore $U+V \subset$ $G_{T^{\varkappa}}(U)=Z$.

Lemma 1.1 Let $1<p^{i}<p \leq r \leq \infty ; 0<|\varkappa| \leq \frac{|\lambda|}{1+a} ; 0<t, \eta \leq T \leq 1 ; 0<\gamma<\gamma_{0}$; $\nu=\left(\nu_{1}, \nu_{2}, \ldots, \nu_{n}\right), \nu_{j} \geq 0$ are integers $(j=1,2, \ldots, n) ; \varphi \in L_{\left.p^{i}\right), \varkappa, a}(G)$ and

$$
\begin{gathered}
m^{i}=\left(l^{i}, \lambda\right)-(\nu, \lambda)-(|\lambda|-|\varkappa|-|\varkappa| a)\left(\frac{1}{p^{i}-\varepsilon}-\frac{1}{p-\varepsilon}\right), \\
(\nu, \lambda)=\sum_{j=1}^{n} \nu_{j} \lambda_{j},|\lambda|=\sum_{i=1}^{n} \lambda_{j} \\
R_{\eta}^{i}(x)=\int_{0}^{\eta} t^{-1-|\lambda|-(\nu, \lambda)+\left(l^{i}, \lambda\right)} d t \int_{\mathbb{R}^{n}} \varphi(x+y) \psi\left(\frac{y}{t^{\lambda}}, \frac{\rho\left(t^{\lambda}, x\right)}{t^{\lambda}}, \rho^{\prime}\left(t^{\lambda}, x\right)\right) d y \\
R_{\eta, T}^{i}(x)=\int_{\eta}^{T} t^{-1-|\lambda|-(\nu, \lambda)+\left(l^{i}, \lambda\right)} d t \int_{\mathbb{R}^{n}} \varphi(x+y) \psi\left(\frac{y}{t^{\lambda}}, \frac{\rho\left(t^{\lambda}, x\right)}{t^{\lambda}}, \rho^{\prime}\left(t^{\lambda}, x\right)\right) d y .
\end{gathered}
$$

Then

$$
\begin{gathered}
\sup _{\bar{x} \in U}\left\|R_{\eta}^{i}\right\|_{p-\varepsilon, U_{\gamma} \varkappa(\bar{x})} \leq C^{1}\|\varphi\|_{\left.p^{i}\right), \varkappa, a, Z} \varepsilon^{-\frac{1}{p^{i}-\varepsilon}} \gamma^{|\varkappa| \frac{a+1}{p^{i}-\varepsilon}} \eta^{m_{i}}\left(m^{i}>0\right), \\
\sup _{\bar{x} \in U}\left\|R_{\eta, T}^{i}\right\|_{p-\varepsilon, U_{\gamma^{\varkappa}}(\bar{x})} \leq C^{2}\|\varphi\|_{\left.p^{i}\right), \varkappa, a, Z^{\varepsilon}} \frac{-\frac{1}{p^{i}-\varepsilon}}{|\varkappa| \frac{a+1}{p^{i}-\varepsilon}} \\
\times\left\{\begin{array}{l}
T^{m^{i}} \text { for } m^{i}>0, \\
\ln \frac{T}{\eta} \text { for } m^{i}=0, \\
\eta^{m^{2}} \text { for } m^{i}<0 .
\end{array}\right.
\end{gathered}
$$

Here $U_{\gamma^{\varkappa}} \bar{x}=\left\{x:\left|x_{j}-\bar{x}_{j}\right|<\frac{1}{2} \gamma^{\varkappa_{j}}, j=1,2, \ldots, n\right.$ and $C^{1}, C^{2}$ are constants independent of $\varphi, \gamma, \eta, T$ and $\varepsilon$.

Proof. Applying the generalized Minkowskii inequality for any $\bar{x} \in U$, we get

$$
\left\|R_{\eta}^{i}\right\|_{p-\varepsilon, U_{\gamma^{\varkappa}}(\bar{x})} \leq C \int_{0}^{\eta_{0}} t^{-1-|\lambda|-(\nu, \lambda)+\left(l^{i}, \lambda\right)}\|\phi\|_{p-\varepsilon, U_{\gamma \varkappa}(\bar{x})} d t,
$$

where

$$
\phi(x, t)=\int_{\mathbb{R}^{n}} \varphi(x+y) \Psi\left(\frac{y}{t^{\lambda}}, \frac{\rho\left(t^{\lambda}, x\right)}{t^{\lambda}}, \rho^{1}\left(t^{\lambda, x}\right)\right) d y .
$$


From Hölder's inequality $(p \leq r)$, we have

$$
\|\phi(., t)\|_{p-\varepsilon, U_{\gamma^{\varkappa}}(\bar{x})} \leq\|\phi(., t)\|_{r-\varepsilon, U_{\gamma^{\varkappa}}(\bar{x})} \gamma^{|\varkappa|\left(\frac{1}{p-\varepsilon}-\frac{1}{r-\varepsilon}\right)} .
$$

Now we estimate the norm $\|\phi(., t)\|_{p-\varepsilon, U_{\gamma \varkappa}(\bar{x})}$.

Let $\chi$ be the characteristic function of $S(\Psi)$ and let $\Psi(x, y, z)$ satisfy condition $|\Psi(x, y, z)|$ $\leq C\left|\Psi_{1}(x)\right|$, for a.e. $(y, z) \in \mathbb{R}^{n} \times \mathbb{R}^{n}$.

Account into that $1<p^{i}<r \leq \infty, s \leq r\left(\frac{1}{s}=1-\frac{1}{p^{i}-\varepsilon}+\frac{1}{r-\varepsilon}\right)$, represent the integrand function (1.11) as the form

$$
\left.\|\varphi \Psi\|=\left(|\varphi|^{p^{i}-\varepsilon}|\Psi|^{\frac{1}{s}}\right)^{\frac{1}{p^{i}-\varepsilon}}\right)\left(|\Psi|^{p^{i}-\varepsilon} \chi\right)^{\frac{1}{p^{i}-\varepsilon}-\frac{1}{r-\varepsilon}}-\left(|\Psi|^{s}\right)^{\frac{1}{s}-\frac{1}{r-\varepsilon}}
$$

and applying Hölders inequality $\left(\frac{1}{r-\varepsilon}+\left(\frac{1}{p^{i}-\varepsilon}-\frac{1}{r-\varepsilon}\right)+\left(\frac{1}{s}-\frac{1}{r-\varepsilon}\right)=1\right)$, we have

$$
\begin{aligned}
& \|\phi(\cdot, t)\|_{r-\varepsilon, U_{\gamma^{\varkappa}}(\bar{x}) \leq} \sup _{\bar{x} \in U_{\gamma^{\varkappa}(\bar{x})}}\left(\int_{\mathbb{R}^{n}}|\varphi(x+y)|^{p^{i}-\varepsilon} \chi\left(\frac{y}{t^{\lambda}}\right) d y\right)^{\frac{1}{p^{i}-\varepsilon}-\frac{1}{r-\varepsilon}} \\
& \quad \times \sup _{\bar{y} \in V}\left(\int_{U_{\gamma^{\varkappa}(\bar{x})}} \mid \varphi(x+y)^{p^{i}-\varepsilon} d x\right)^{\frac{1}{p^{i}-\varepsilon}}\left(\int_{\mathbb{R}^{n}}\left|\Psi_{1}\left(\frac{y}{t^{\lambda}}\right)\right|{ }^{s} d y\right)^{\frac{1}{s}} .
\end{aligned}
$$

For any $x \in U$, we have

$$
\begin{gathered}
\int|\varphi(x+y)|^{p^{i}-\varepsilon} \chi\left(\frac{y}{t^{\lambda}}\right) d y \leq \int_{Z_{t^{\lambda}}(x)}|\varphi(y)|^{p^{i}-\varepsilon} d y \\
\leq \int_{Z_{t^{\varkappa}}(x)}|\varphi(y)|^{p^{i}-\varepsilon} d y \leq\|\varphi\|_{p^{i}-\varepsilon, Z_{t^{\lambda}}(x)}^{p^{i}-\varepsilon} \leq\|\varphi\|_{p), \varkappa, a ; Z}^{p^{i}-\varepsilon} \varepsilon^{-1} \gamma^{|\varkappa|(a+1)} .
\end{gathered}
$$

Also, for $y \in U$

$$
\begin{aligned}
\int_{U_{\gamma^{\varkappa}(\bar{x})}}|\varphi(x+y)|^{p^{i}-\varepsilon} d x \leq & \int_{U_{\gamma^{\varkappa}}(\bar{x}+y)}|\varphi(x)|^{p^{i}-\varepsilon} d x \leq\|\varphi\|_{p), \varkappa, a ; Z^{i}}^{p^{i}-\varepsilon} \gamma^{-1} \gamma^{|\varkappa|(a+1)}, \\
& \int_{\mathbb{R}^{n}}\left|\Psi\left(\frac{y}{t^{\lambda}}\right)\right|^{s} d y=t^{|\lambda|}\left\|\Psi_{1}\right\|_{s}^{s} .
\end{aligned}
$$

From inequalities (1.12)-(1.16) for $(r=p)$ it is implies that

$$
\begin{aligned}
& \|\phi(\cdot, t)\|_{p-\varepsilon, U_{\gamma^{\varkappa}}(\bar{x})}
\end{aligned}
$$

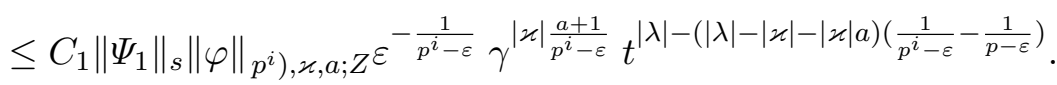

Take into account last inequality in (1.10) for all $x \in U$, we have

$$
\left\|R_{\eta}^{i}\right\|_{p-\varepsilon, U_{\gamma^{\varkappa}}(\bar{x})} \leq C^{1}\|\varphi\|_{\left.p^{i}\right), \varkappa, a ; Z} \varepsilon^{-\frac{1}{p^{i}-\varepsilon}} \gamma^{|\varkappa| \frac{a+1}{p^{i}-\varepsilon}} \eta^{m^{i}}\left(m^{i}>0\right) .
$$

Similarly, we can prove (1.9).

This complete the proof. 


\section{Main results}

Now we proved theorems for functions from grand generalized Sobolev-Morrey spaces $\bigcap_{i=0}^{n} L_{\left.p^{i}\right), \varkappa, a}^{<i^{i}>}(G)$.

Theorem 2.1 Let $G \subset \mathbb{R}^{n}$ be an open bounded set satisfying the flexible $\lambda$-horn condition (see [1]); $1<p^{i}<p \leq \infty ;|\varkappa| \leq \frac{|\lambda|}{1+a} ; \nu=\left(\nu_{1}, \nu_{2}, \ldots, \nu_{n}\right), \nu_{j} \geq 0$ are integers $(j=1,2, \ldots, n) ; m^{i}>0(i=1,2, \ldots, n)$, and $f \in \bigcap_{i=0}^{n} L_{\left.p^{i}\right), \varkappa, a}^{<l^{i}>}(G)$.

Then the following embeddings hold

$$
D^{\nu}: \bigcap_{i=0}^{n} L_{\left.p^{i}\right), \varkappa, a}^{<l^{i}>}(G) \hookrightarrow L_{p-\varepsilon}(G) .
$$

More precisely, for $f \in \bigcap_{i=0}^{n} L_{\left.p^{i}\right), \varkappa, a}^{<l^{i}>}(G)$ there exists a generalized (weak) derivatives $D^{\nu} f$ and

$$
\left\|D^{\nu} f\right\|_{p-\varepsilon, G} \leq C^{1}(\varepsilon) \sum_{i=0}^{n} T^{m^{i}}\left\|D^{l^{i}} f\right\|_{\left.p^{i}\right), \varkappa, a ; G} .
$$

In particular, if $m^{i, 0}=\left(l^{i}, \lambda\right)-(\nu, \lambda)-(|\lambda|-|\varkappa|-|\varkappa| a) \frac{1}{p^{i}-\varepsilon}>0(i=0,1,2, \ldots, n)$, then $D^{\nu} f$ are continuous on $G$ and

$$
\sup _{x \in G}\left|D^{\nu} f(x)\right| \leq C^{1}(\varepsilon) \sum_{i=0}^{n} T^{m^{i}, 0}\left\|D^{l^{i}} f\right\|_{\left.p^{i}\right), \varkappa, a ; G},
$$

where $0<T \leq d_{0}, C^{1}(\varepsilon)=C^{1} \varepsilon^{-\frac{1}{p^{i}-\varepsilon}}$ and $C^{1}$ is a constant independent of $f, T$ and $\varepsilon$.

Proof. At first note that in the conditions of Theorem 2.1 there exists a generalized derivatives $D^{\nu} f$ on $G$. Indeed, from the condition $m^{i}>0(i=1, \ldots, n)$ it follows that for $p^{i}<p,|\varkappa| \leq \frac{|\lambda|}{1+a}$, and

$$
\bigcap_{i=0}^{n} L_{\left.p^{i}\right), \varkappa, a(G)}^{<l^{i}>} \hookrightarrow \bigcap_{i=0}^{n} L_{\left.p^{i}\right)(G)}^{<l^{i}>} \hookrightarrow \bigcap_{i=0}^{n} L_{p^{i}-\varepsilon}^{<l^{i}>}(G)\left(p^{i}-\varepsilon>1\right) .
$$

Then $D^{\nu} f$ exists on $G$ and belongs to $L_{p^{i}-\varepsilon}(G)$ and for almost each $x \in G$ the integral identity is hold

$$
\begin{gathered}
D^{\nu} f(x)=f_{T^{\lambda}}^{(\nu)}(x)+\int_{0}^{T} \int_{\mathbb{R}^{n}} \sum_{i=1}^{n} t^{-1-|\lambda|-(\nu, \lambda)+\left(l^{i}, \lambda\right)} D^{l^{i}} f(x+y) \\
\times M_{i}^{(\nu)}\left(\frac{y}{t^{\lambda}}, \frac{\rho\left(t^{\lambda}, x\right)}{t^{\lambda}}, \rho^{\prime}\left(t^{\lambda}, x\right)\right) d y d t \\
f_{T^{\lambda}}^{(\nu)}(x)=(-1)^{|\nu|} T^{-|\lambda|-(\nu, \lambda)+\left(l^{0}, \lambda\right)} \\
\times \int_{\mathbb{R}^{n}} D^{l^{0}} f(x+y) \Omega^{(\nu)}\left(\frac{y}{T^{\lambda}}, \frac{\rho\left(T^{\lambda}, x\right)}{T^{\lambda}}, \rho^{\prime}\left(T^{\lambda}, x\right)\right) d y
\end{gathered}
$$


where $0<T \leq \min \left(d_{0}, T_{0}\right), \Omega(\cdot, y, z)$ and $M_{i}(\cdot, y, z) \in C_{0}^{\infty}\left(\mathbb{R}^{n}\right)$ (see, [9]). Recall that the support of the integral representations (2.3) and (2.4) is $V(\lambda)$. Applying the Minkowskii inequality, from (2.3) and (2.4), we get

$$
\left\|D^{\nu} f\right\|_{p-\varepsilon, G} \leq\left\|f_{T^{\lambda}}^{\nu}\right\|_{p-\varepsilon, G}+\sum_{i=1}^{n}\left\|R_{T}^{i}\right\|_{p-\varepsilon, G}
$$

By (1.8) for $U=G, D^{l^{0}} f=\phi, \Psi=\Omega^{(\nu)}, \eta=T$, one has

$$
\left\|f_{T^{\lambda}}^{\nu}\right\|_{p-\varepsilon, G} \leq C_{1}(\varepsilon)\left\|D^{l^{0}} f\right\|_{\left.p^{0}\right), \varkappa, a, G} T^{m^{0}}
$$

and for $U=G, D^{l^{i}}=\phi, \Psi=M_{i}^{(\nu)}, \eta=T$, we have

$$
\left\|R_{T}^{i}\right\|_{p-\varepsilon, G} \leq C_{2}(\varepsilon)\left\|D^{l^{i}} f\right\|_{\left.p^{i}\right), \varkappa, a ; G} T^{m^{i}} .
$$

Substituting (2.6) and (2.7) in (2.5), we get (2.5).

Let $m^{i, 0}>0(i=1,2, \ldots, n)$. By (2.3), (2.4) from inequality (2.5) for $p=\infty$ and $m^{i}(p=\infty)=m^{i, 0}(i=1, \ldots, n)$, we obtain

$$
\left\|D^{\nu} f-f_{T^{\lambda}}^{\nu}\right\|_{\infty, G} \leq \sum_{i=1}^{n} T^{m^{i, 0}}\left\|D^{l^{i}} f\right\|_{\left.p^{i}\right), \varkappa, a ; G} .
$$

It follows that the left hand side of the last inequality tends to zero as $T \rightarrow 0$. Since $f_{T^{\lambda}}^{\nu}$ is continuous on $G$, the convergence in $L_{\infty}(G)$ coincides with usually uniform convergence. Then the limit function $D^{\nu} f$ is continuous on $G$.

Theorem 2.1 is proved.

Let $\rho$ be an $n$-dimensional vector.

Theorem 2.2 Let all the conditions of Theorem 2.1 be fulfilled. If $m^{i}>0(i=1,2, \ldots, n)$, then $D^{\nu} f$ satisfies the Hölder condition with exponent $\sigma$ on $G$ in the metric of $L_{p-\varepsilon}$. More exactly,

$$
\left\|\Delta(\zeta, G) D^{\nu} f\right\|_{p-\varepsilon, G} \leq C^{2}(\varepsilon)\|f\|_{\bigcap_{i=0}^{n} L_{\left.p^{i}\right), \varkappa, a}^{<l^{i}>}(G)}|\zeta|^{\sigma}
$$

where $C^{2}(\varepsilon)=C^{2} \varepsilon^{-\frac{1}{p^{i}-\varepsilon}}$ and $C^{2}$ is a constant independent of $f$ and $\varepsilon$ and $\sigma$ is an arbitrary number satisfying the inequalities:

$$
\begin{gathered}
0 \leq \sigma \leq 1, \text { if } \frac{m^{0}}{\lambda_{0}}>0, \\
0 \leq \sigma<1, \text { if } \frac{m^{0}}{\lambda_{0}}=1, \\
0 \leq \sigma \leq \frac{m^{0}}{\lambda_{0}}, \text { if } \frac{m^{0}}{\lambda_{0}}<1,
\end{gathered}
$$

where $m^{0}=\min _{1 \leq i \leq n} m^{i} ; \lambda_{0}=\max _{1 \leq i \leq n} \lambda_{i}$.

If $m^{i, 0}>0(i=1,2, \ldots, n)$, then

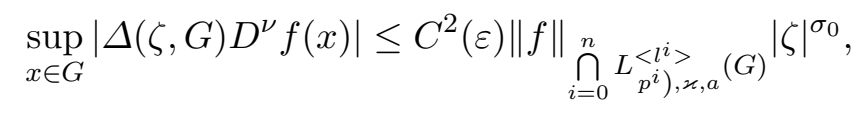

where $\sigma_{0}$ satisfy the same conditions as $\sigma$ with $m^{i, 0}$ instead of $m^{i}$. 
Proof. According to Lemma 8.6 in [1], there exists a domain $G_{\sigma} \subset G(\sigma=\xi r(x), r=$ $\left.\rho_{\lambda}(x, \partial G), x \in G\right)$.

Suppose that $|\zeta|_{\lambda}<\sigma$, then for any $x \in G_{\sigma}$ the segment joining the points $x, x+\zeta$ is contained in $G$. Consequently, for all the points of this segment, identities (2.3) and (2.4) with the same kernels are valid. After same transformations, from (2.3) and (2.4), we get

$$
\begin{aligned}
& \left|\Delta(\zeta, G) D^{\nu} f(x)\right| \leq T^{-|\lambda|-(\nu, \lambda)+\left(l^{0}, \lambda\right)} \\
& \times \int_{\mathbb{R}^{n}} D^{l^{0}} f(x+y) \mid \Omega^{(\nu)}\left(\frac{y-\zeta}{T^{\lambda}}, \frac{\rho\left(T^{\lambda}, x\right)}{T^{\lambda}}, \rho^{\prime}\left(T^{\lambda}, x\right)\right) \\
& -\Omega^{(\nu)}\left(\frac{y}{T^{\lambda}}, \frac{\rho\left(T^{\lambda}, x\right)}{T^{\lambda}}, \rho^{\prime}\left(T^{\lambda}, x\right)\right) \mid d y \\
& +\sum_{i=1}^{n} \int_{0}^{|\zeta|^{\frac{1}{\lambda_{0}}}} t^{-1-|\lambda|-(\nu, \lambda)+\left(l^{i}, \lambda\right)} \int_{\mathbb{R}^{n}}\left(\left|D^{l^{i}} f(x+y+\zeta)\right|+\left|D^{l^{i}} f(x+y)\right|\right) \\
& \times \mid M_{i}^{(\nu)}\left(\frac{y}{t^{\lambda}}, \frac{\rho\left(t^{\lambda}, x\right)}{t^{\lambda}}, \rho^{\prime}\left(t^{\lambda}, x\right) \mid d y d t\right. \\
& +\int_{|\zeta|^{\frac{1}{\lambda_{0}}}}^{T} t^{-1-|\lambda|-(\nu, \lambda)+\left(l^{i}, \lambda\right)} \int_{\mathbb{R}^{n}}\left|D^{l^{i}} f(x+y)\right| \mid M_{i}^{(\nu)}\left(\frac{y-\zeta}{t^{\lambda}}, \frac{\rho\left(t^{\lambda}, x\right)}{t^{\lambda}}, \rho^{\prime}\left(t^{\lambda}, x\right)\right) \\
& -M_{i}^{(\nu)}\left(\frac{y}{t^{\lambda}}, \frac{\rho\left(t^{\lambda}, x\right)}{t^{\lambda}}, \rho^{\prime}\left(t^{\lambda}, x\right)\right) \mid d y d t \\
& =R(x, \zeta)+\sum_{i=0}^{n}\left(R_{1, i}(x, \zeta)+R_{2, i}(x, \zeta)\right),
\end{aligned}
$$

where $0<T \leq d_{0}$. We also assume that $|\zeta|_{\lambda}<T$, and consequently $|\zeta|_{\lambda} \leq \min \{\sigma, T\}$. If $x \in G \backslash G_{\sigma}$, then $\Delta(\zeta, G) D^{\nu} f(x)=0$. By $(2.10)$

$$
\begin{gathered}
\left\|\Delta(\zeta, G) D^{\nu} f\right\|_{p-\varepsilon, G}=\left\|\Delta(\zeta, G) D^{\nu} f\right\|_{p-\varepsilon, G_{\sigma}} \\
\leq\|R(\cdot, \zeta)\|_{p-\varepsilon, G_{\sigma}}+\sum_{i=0}^{n}\left(\left\|R_{1, i}(\cdot, \zeta)\right\|_{p-\varepsilon, G_{\sigma}}+\left\|R_{2, i}(\cdot, \zeta)\right\|_{p-\varepsilon, G_{\sigma}}\right) .
\end{gathered}
$$

Note that

$$
\begin{gathered}
\left|\Omega^{(\nu)}\left(\frac{y-\zeta}{T^{\lambda}}, \frac{\rho\left(T^{\lambda}, x\right)}{T^{\lambda}}, \rho^{\prime}\left(T^{\lambda}, x\right)\right)-\Omega^{(\nu)}\left(\frac{y}{T^{\lambda}}, \frac{\rho\left(T^{\lambda}, x\right)}{T^{\lambda}}, \rho^{\prime}\left(T^{\lambda}, x\right)\right)\right| \\
\quad \leq \sum_{i=0}^{n} T^{-\lambda_{j}} \int_{0}^{|\zeta|}\left|D_{j} \Omega^{(\nu)}\left(\frac{y-\xi e_{\zeta}}{T^{\lambda}}, \frac{\rho\left(T^{\lambda}, x\right)}{T^{\lambda}}, \rho^{\prime}\left(T^{\lambda}, x\right)\right)\right| d \xi
\end{gathered}
$$

$e_{\zeta}=\frac{\zeta}{|\zeta|}$. Therefore

$$
\begin{gathered}
R(x, \zeta) \leq \sum_{j=1}^{n} T^{-\lambda_{j}-|\lambda|-(\nu, \lambda)+\left(l^{i}, \lambda\right)} \\
\times \int_{0}^{|\zeta|} d \xi \int_{\mathbb{R}^{n}}\left|D^{l^{0}} f\left(x+\xi e_{\zeta}+y\right)\right|\left|D_{j} \Omega^{(\nu)}\left(\frac{y}{T^{\lambda}}, \frac{\rho\left(T^{\lambda,} x\right)}{T^{\lambda}}, \rho^{\prime}\left(T^{\lambda}, x\right)\right)\right| d y .
\end{gathered}
$$


Similarly, we get

$$
\begin{gathered}
R_{2, i}(x, \zeta) \leq \sum_{j=1}^{n} \int_{0}^{|\zeta|} t^{-1-|\lambda|-(\nu, \lambda)-\lambda_{j}+\left(l^{i}, \lambda\right)} d t \\
\times \int_{\mathbb{R}^{n}}\left|D^{l^{i}} f\left(x+\xi e_{\zeta}+y\right)\right|\left|D_{j} M_{i}^{\nu}\left(\frac{y}{t^{\lambda}}, \frac{\rho\left(t^{\lambda}, x\right)}{t^{\lambda}}, \rho^{\prime}\left(t^{\lambda}, x\right)\right)\right| d y .
\end{gathered}
$$

Taking into account $\xi e_{\zeta}+G_{\sigma} \subset G$, by inequality (1.8) for $U=G, D^{l^{0}} f=\phi$, $\Omega^{(\nu)}=\psi$, and $p^{i}=p^{0}$ one has

$$
\|R(\cdot, \zeta)\|_{p-\varepsilon, G_{\delta}} \leq C_{1}(\varepsilon)\|\zeta \mid\| D^{l^{0}} f \|_{\left.p^{0}\right), \varkappa, a ; G} .
$$

By (1.9) for $U=G, t=T, D^{l^{i}} f=\phi, M_{i}^{\nu}=\Psi, \eta=|\zeta|^{\frac{1}{\lambda_{0}}}$, we have

$$
\left\|R_{1, i}(\cdot, \zeta)\right\|_{p-\varepsilon, G_{\sigma}} \leq C_{2}(\varepsilon)|\zeta|^{\frac{m^{i}}{\lambda_{0}}}\left\|D^{l^{i}} f\right\|_{\left.p^{i}\right), \varkappa, a ; G} \cdot
$$

Also from (1.10) for $U=G, \eta=|\zeta|^{\frac{1}{\lambda_{0}}}, D^{l^{i}} f=\varphi, M^{(\nu)}=\psi$, we have

$$
\left\|R_{2, i}(\cdot, \zeta)\right\|_{p-\varepsilon, G_{\sigma}} \leq C_{2}(\varepsilon)|\zeta|^{\sigma}\left\|D^{l^{i}} f\right\|_{\left.p^{i}\right), \varkappa, a ; G} \cdot
$$

It follows from (2.11) - (2.14) that

$$
\left\|\Delta(\zeta, G) D^{\nu} f\right\|_{p-\varepsilon, G_{\sigma}} \leq C_{3}(\varepsilon)\|f\|_{\bigcap_{i=0}^{n} L_{\left.p^{i}\right), \varkappa, a}^{<l^{i}>}(G)}|\zeta|^{\sigma} \mid .
$$

Estimating $\left\|D^{\nu} f\right\|_{p-\varepsilon, G}$ by means of (2.1), in this case we get estimation (2.8).

This complete the proof.

\section{References}

1. Besov O.V., Ilyin V.P., Nikolskii S.M.: Integral representations of functions and embeddings theorems, M. Nauka, 480 p. (1996).

2. Fiorenza A., Karadzhov C.E.: Grand and small Lebesgue spaces and their analogs, J. Anal. Appl. 23 (4) 657-681 (2004)

3. Iwaniec T., Sbordone C.: On the integrability of the Jacobian under minimal hypotheses, Arch. Ration. Mech. Anal. 119 129-143 (1992).

4. Kokilashvili V.: The Riemann boundary value problem for analytic functions in the frame of grand $L_{p}$ spaces, Bull. Georgian Nat. Acad. Sci. 4 (1) 5-7 (2010).

5. Kokilashvili V., Meskhi A.: Trace inequalities for fractional integrals in grand Lebesgue spaces, Studia Math. 210 (2), 159-176 (2012)

6. Kokilashvili V., Meskhi A., Rafeiro H.: Estimates for nondivergence elliptic equations with VMO coefficients in generalized grand Morrey spaces, Complex Var. Elliptic Equ. 8 (59), 1169-1184 (2014)

7. Meskhi A.: Maximal functions, potentials and singular integrals in grand Morrey spaces, Complex Var. Elliptic Equ. 534-793 (2011) http://dx.doi.org/10.1080/17476933:2010.

8. Mizuta Y., Ohno T.: Trudingers exponential integrability for Riesz potensials of function in generalized grand Morrey spaces, J. Math. Anal. Appl. 420 (1), 268-278 (2014).

9. Najafov A.M., Kadimova L.Sh.: Theorems on imbedding of functions from the SobolevMorrey generalized space, Proc. A. Razmadze Math. Inst. 154, 97-109 (2010). 
10. Najafov A.M.: The differential properties of functions from Sobolev-Morrey type spaces of fractional order, J. Math. Res., 7 (3), 149-158 (2015).

11. Najafov A.M., Orujova A.T.: On the solution of a class of partial differential equations, Electron. J. Qual. Theory Differ. Equ. 2017 (44), 1-9 (2017).

12. Najafov A.M., Babayev R.F.: Some properties of functions from generalized SobolevMorrey type spaces, Math. Aeterna, 7 (3), 301-311 (2017).

13. Najafov A.M., Rustamova N.R.: Some differential properties of anisotropic grand Sobolev-Morrey spaces, Trans. A. Razmadze Math. Inst., 172 (1), $82-89$ (2018).

14. Najafov A.M.: On embedding theorems in small small Sobolev-Morrey spaces, Inter. Conf. "Modern problems of Math. and Mech."devoted to the 60th anniversary of the Inst. Math. and Mech., 23-25 October, Baku, Azerbaijan, p. 403.

15. Najafov A.M., Alekberli S.T.: On properties functions from grand grand SobolevMorrey spaces, J. Baku Eng. Univ., 2 (1), (2018).

16. Najafov A.M., Gasimova A.M.: On embedding theorems in grand grand NikolskiiMorrey spaces, Eur. J. Pure Appl. Math., 12 (4), 1602-1611 (2019)

17. Najafov A.M., Rustamova N.R., Alekberli S.T.: On solvability of a quasi-elliptic partial differential equations. J. Elliptic Parabol. Equ., On line version, 1-13 (2019)

18. Najafov A.M., Alekberli S.T.: Some properties of grand Sobolev-Morrey spaces with dominant mixed derivatives, J. Math. Inequal. 13 (4), 1171-1180 (2019).

19. Samko S.G., Umarkhadzhiev S.M., On Iwaniec - Sbordone spaces on sets which may have infinite measure, Azerb. J. Math. 1 (1), 67-84 (2011)

20. Samko S.G., Umarkhadzhiev S.M.: On Iwaniec - Sbordone spaces on sets which may have infinite measure, Azerb. J. Math. 1 (2), 143-144 (2011)

21. Sbordone C.: Grand Sobolev spaces and their applications to variational problems, in: Le Mathematiche, LI, 335-347 ( 1996) Fasc. II. 